\title{
3D plasmonic nanoarchitecture as an emerging biosensing platform
}

\author{
Md Masud Parvez Arnob \& Wei-Chuan Shih*,1,2,3,4 \\ ${ }^{1}$ Department of Electrical \& Computer Engineering, University of Houston, Houston, TX 77204, USA \\ ${ }^{2}$ Department of Biomedical Engineering, University of Houston, Houston, TX 77204, USA \\ ${ }^{3}$ Program of Materials Science \& Engineering, University of Houston, Houston, TX 77204, USA \\ ${ }^{4}$ Department of Chemistry, University of Houston, Houston, TX 77204, USA \\ * Author for correspondence: wshih@uh.edu
}

First draft submitted: 14 August 2017; Accepted for publication: 18 August 2017; Published online: 10 October 2017

\section{Propagating \& localized surface plasmon resonance}

Recent advances in plasmonics of coinage metals have attracted significant interest in the biosensing community $[1,2]$. Surface plasmon resonance refers to the oscillation of conduction band electrons excited by light. Often touted as a label-free sensing technique, surface plasmon resonance based sensing and imaging have become commercially available for quite some time, and are employed in applications such as studying protein-protein interactions, immunochemical procedures and drug-receptor binding. Traditional surface plasmon resonance sensors rely on the propagating surface plasmon resonance (PSPR), where light-electron coupling is obtained via total-internalreflection, a condition typically achieved by prism coupling [3-5]. Due to the unconventional nature of light coupling, PSPR sensors face challenges in instrument miniaturization and simplification. Another limiting factor for PSPR imaging is spatial resolution. Although, confined to the metal-dielectric interface, plasmon waves do propagate along the interface, therefore degrades spatial resolution.

Localized surface plasmon resonance (LSPR) refers to nonpropagating plasmon modes identified in various nanostructures and colloidal nanoparticles [6]. In contrast to PSPR, LSPR provides the possibility of free-space light coupling and highly confined fields in all three dimensions, thanks to the nanostructures. These features can significantly reduce system complexity and measurement requirements [7]. In addition, since nanostructures are involved, the latitude of engineering design has substantially increased compared with PSPR where a thin film suffices.

\section{LSPR associated light concentration \& field enhancement}

The light concentrating property of LSPR is a focal point of modern plasmonic biosensing research. Typically understood as collective electron oscillation, LSPR produces highly localized electromagnetic field enhancement in plasmonic nanomaterials [6]. Plasmonic hot-spots refer to the locations, in close proximity of nanostructures, where electromagnetic fields are particularly enhanced relative to the incident field. LSPR associated local field concentration has been shown to enhance a variety of electronic as well as vibrational spectroscopic techniques. Among those, prominent examples can be drawn from Raman scattering, infrared and near-infrared (NIR) absorption and fluorescence [8-11].

Traditional plasmonic nanomaterials are 1D (e.g., colloidal nanoparticles) or 2D (lithographically patterned nanostructure arrays) in nature, which typically result in sparse field concentration patterns. To improve efficiency and better utilization of hot-spots, the concept of 3D plasmonic nanoarchitecture, where abundant hot-spots are formed in a 3D volumetric fashion, have emerged [12]. Practically, there are several potential advantages of plasmonic sensing. First, many plasmonic sensing mechanisms can be implemented in a label-free fashion which requires no additional binding of fluorescent tag or radioisotope [13]. Plasmonic sensors work naturally with optical interrogation technology and therefore can be non- or minimally-invasive. In this Editorial, we introduce three distinct 3D plasmonic nanoarchitecture for LSPR-based biosensing. A key feature shared by the three examples is no lithography is involved in the formation of hot-spots. We also comment on their figure of merit from practical aspects such as performance, robustness and cost-effectiveness. 


\section{Nanoporous gold nanoparticles \& arrays}

Nanoporous gold (NPG) nanoparticles and arrays can be formed by a hybrid fabrication process combining top-down lithography and bottom-up atomic dealloying [14,15]. The lithography step patterns gold-silver film into nanoparticles of well-defined shape and size, while the dealloying step produces highly porous nanostructures throughout each individual nanoparticles. Single NPG nanoparticle features large surface area, high-density plasmonic hot-spots, and tunable plasmonic resonance [16,17]. Due to the less metallic nature, single NPG nanoparticles feature LSPR resonance in the NIR wavelength range from $\sim 700$ to $1800 \mathrm{~nm}$ depending on its diameter and pore size [15]. NPG nanoparticle arrays, in contrast, feature blue-shifted LSPR due to far-field plasmonic coupling among nanoparticles [18]. The degree of blueshift is also highly tunable by diameter and inter-particle distance. Recently, nanocomposites formed by colloidal AuNP on NPG disks have been shown to form additional hot-spots at the binding sites [19]. NPG arrays have been demonstrated in various plasmonic sensing mechanisms such as refractive index sensing via extinction spectroscopy, as well as spectroscopic fingerprinting by enhanced fluorescence, NIR absorption, and Raman spectroscopy [8-11,14]. A number of label-free biosensing applications have been developed in the past 4 years for nucleic acids, proteins, enzymes, metabolites, neurotransmitters, polycyclic aromatic hydrocarbons, carcinogenic environmental and food contaminants, and etc [11,19-23].

\section{Stacks of orthogonally arranged nanowires}

Using solvent-assisted nanotransfer printing (S-nTP), vertically stacked 3D cross-point plasmonic structures have been made [24]. A key advantage of the fabrication technique lies in the sequential additive printing on nontraditional substrates such as soft materials. The design of $3 \mathrm{D}$ crossbar array is analogous to recent memory device architectures designed for maximizing information storage density and improve device performance [25]. The S-nTP printed nanowire stacks can provide both in-plane in the nanogap region as well as out-of-plane coupling at the cross-points where two orthogonal nanowires meet. The latter, in particular, facilitates the formation of plasmonic hot-spots by geometrical constraints. By area mapping, the plasmonic substrate appears to exhibit high uniformity, an important attribute for biosensing. The cross-point structures have been successfully printed on contact lens and glucose sensing at physiologically relevant concentrations has been demonstrated. The proof-of principle results show the potential of glucose sensing in tears by optically interrogating the contact lens, and perhaps other biomarkers in bodily fluids.

\section{Gold-coated eggshells}

Recently, researchers have been inspired by naturally occurring micro/nanostructures to develop simple, low-cost and green nanotechnology. Among those, eggshells have been demonstrated to provide a versatile template for building 3D plasmonic nanostructures with extremely low-cost [26]. By coating a thin layer of gold film on different eggshell regions including the outer and inner shells and the membrane, these daily waste materials have been employed in sensing bacteria with surprisingly good sensitivity down to single cells [27]. The outer shells have been shown to provide the best sensitivity and good uniformity. Such a technology can potentially be applied to label-free pathogen detection and typing without time-consuming cell culture, which is the gold standard for diagnostics.

\section{Comparison \& future perspective}

Although, artificial lithography can pattern sub-10 nm features as plasmonic hot-spots, it is difficult, if not impossible, to scale up. In this Editorial, we have reviewed three recent examples for lithography-free hot-spot formation. Cross-points nanowire stacks resemble modern memory architectures for high capacity and efficiency, and can be printed on soft substrates by sequential addition. The underlying fabrication technique, S-nTP, is powerful yet costly. Gold-coated eggshells, in contrast, represent an extremely low-cost alternative for bacteria sensing and identification. NPG nanoparticles have been shown to be the most versatile in various applications, and are cost-effective. 3D plasmonic nanoarchitectures are an interesting class of novel nanomaterials. Their most important biosensing advantages lie in label-free, minimal or no sample preparation, low invasiveness and optical readout. The fact that they can be fabricated on soft substrate suggests their potential use in flexible and wearable devices. The simplicity in both sensor and detector design would lead to cost-effective point-of-care devices.

Supplementary data

To view the supplementary data that accompany this paper please visit the journal website at: www.futuremedicine.com/doi/full/10.2217/nnm-2017-0258 
Financial \& competing interests disclosure

The authors have no relevant affiliations or financial involvement with any organization or entity with a financial interest in or financial conflict with the subject matter or materials discussed in the manuscript. This includes employment, consultancies, honoraria, stock ownership or options, expert testimony, grants or patents received or pending, or royalties.

No writing assistance was utilized in the production of this manuscript

\section{References}

1 Anker JN, Hall WP, Lyandres O, Shah NC, Zhao J, Van Duyne RP. Biosensing with plasmonic nanosensors. Nat. Mater. 7(6), 442-453 (2008).

2 Zeng S, Yong K-T, Roy I, Dinh X-Q, Yu X, Luan F. A review on functionalized gold nanoparticles for biosensing applications. Plasmonics 6(3), 491 (2011).

3 Arwin H, Welin S, Lundström I. Reflectance method for immunoassay on solid surfaces. In: Nonisotopic Immunoassay. Springer, 313-330 (1988).

4 Buckle P, Davies R, Kinning T et al. The resonant mirror: a novel optical sensor for direct sensing of biomolecular interactions part II: applications. Biosensors Bioelectron. 8(7-8), 355-363 (1993).

5 Wood S. DNA-DNA hybridization in real time using BIAcore. Microchem. J. 47(3), 330-337 (1993).

6 Willets KA, Van Duyne RP. Localized surface plasmon resonance spectroscopy and sensing. Annu. Rev. Phys. Chem. 58 267-297 (2007).

7 Hoa X, Kirk A, Tabrizian M. Towards integrated and sensitive surface plasmon resonance biosensors: a review of recent progress. Biosensors Bioelectron. 23(2), 151-160 (2007).

8 Qi J, Motwani P, Gheewala M, Brennan C, Wolfe JC, Shih W-C. Surface-enhanced Raman spectroscopy with monolithic nanoporous gold disk substrates. Nanoscale 5(10), 4105-4109 (2013).

9 Shih W-C, Santos GM, Zhao F, Zenasni O, Arnob MMP. Simultaneous chemical and refractive index sensing in the 1-2.5 $\mu \mathrm{m}$ near-infrared wavelength range on nanoporous gold disks. Nano Lett. 16(7), 4641-4647 (2016).

10 Santos GM, Zhao F, Zeng J, Li M, Shih WC. Label-free, zeptomole cancer biomarker detection by surface-enhanced fluorescence on nanoporous gold disk plasmonic nanoparticles. J. Biophotonics 8(10), 855-863 (2015).

11 Li M, Du Y, Zhao F, Zeng J, Mohan C, Shih W-C. Reagent- and separation-free measurements of urine creatinine concentration using stamping surface enhanced Raman scattering (S-SERS). Biomed. Opt. Express 6(3), 849-858 (2015).

12 Kuzyk A, Schreiber R, Fan Z et al. DNA-based self-assembly of chiral plasmonic nanostructures with tailored optical response. arXiv preprint arXiv. 1108, 3752 (2011).

13 Kabashin A, Evans P, Pastkovsky S et al. Plasmonic nanorod metamaterials for biosensing. Nat. Mater. 8(11), 867 (2009).

14 Zhao F, Zeng J, Arnob MMP et al. Monolithic NPG nanoparticles with large surface area, tunable plasmonics, and high-density internal hot-spots. Nanoscale 6(14), 8199-8207 (2014).

15 Arnob MMP, Zhao F, Li J, Shih W-C. EBL-based fabrication and different modeling approaches for nanoporous gold nanodisks. ACS Photonics 4(8), 1870-1878 (2017).

16 Zeng J, Zhao F, Qi J et al. Internal and external morphology-dependent plasmonic resonance in monolithic nanoporous gold nanoparticles. RSC Adv. 4(69), 36682-36688 (2014).

17 Arnob MMP, Zhao F, Zeng J, Santos GM, Li M, Shih W-C. Laser rapid thermal annealing enables tunable plasmonics in nanoporous gold nanoparticles. Nanoscale 6(21), 12470-12475 (2014).

18 Zhao F, Arnob MMP, Zenasni O, Li J, Shih W-C. Far-field plasmonic coupling in 2-dimensional polycrystalline plasmonic arrays enables wide tunability with low-cost nanofabrication. Nanoscale Horizons 2, 267-276 (2017).

19 Zhao F, Zeng J, Shih W-C. Nanoporous gold nanocomposites as a versatile platform for plasmonic engineering and sensing. Sensors 17(7), 1519 (2017)

20 Qi J, Zeng J, Zhao F et al. Label-free, in situ SERS monitoring of individual DNA hybridization in microfluidics. Nanoscale 6(15), $8521-8526$ (2014).

21 Li M, Zhao F, Zeng J, Qi J, Lu J, Shih W-C. Microfluidic surface-enhanced Raman scattering sensor with monolithically integrated nanoporous gold disk arrays for rapid and label-free biomolecular detection. J. Biomed. Optics 19(11), 111611-111611 (2014).

22 Qiu S, Zhao F, Zenasni O, Li J, Shih W-C. Nanoporous gold disks functionalized with stabilized G-quadruplex moieties for sensing small molecules. ACS Appl. Mater. Interfaces 8(44), 29968-29976 (2016).

23 Qiu S, Zhao F, Zenasni O, Li J, Shih W-C. Catalytic assembly of DNA nanostructures on a nanoporous gold array as 3D architectures for label-free telomerase activity sensing. Nanoscale Horizons 2, 217-224 (2017).

24 Jeong JW, Arnob MMP, Baek KM, Lee SY, Shih WC, Jung YS. 3D cross-point plasmonic nanoarchitectures containing dense and regular hot spots for surface-enhanced raman spectroscopy analysis. Adv. Mater. 28(39), 8695-8704 (2016). 
Editorial Masud Parvez Arnob \& Shih

25 Green JE, Choi JW, Boukai A et al. A 160-kilobit molecular electronic memory patterned at 1011 bits per square centimetre. Nature 445(7126), 414 (2007).

26 Zhou J, Wang S, Nie F, Feng L, Zhu G, Jiang L. Elaborate architecture of the hierarchical hen's eggshell. Nano Res. 4(2), 171-179 (2011).

27 Arnob MMP, Shih W-C. 3-dimensional plasmonic substrates based on chicken eggshell bio-templates for SERS-based bio-sensing. Micromachines-Basel 8(6), 196 (2017). 